\title{
Attention-based distributed speech enhancement for unconstrained microphone arrays with varying number of nodes
}

\author{
Nicolas Furnon \\ Université de Lorraine, CNRS, Inria, Loria \\ F-54000 Nancy, France \\ nicolas.furnon@loria.fr
}

\author{
Slim Essid \\ LTCI, Télécom Paris, Institut Polytechnique de Paris \\ Palaiseau, France
}

\author{
Romain Serizel \\ Université de Lorraine, CNRS, Inria, Loria \\ F-54000 Nancy, France
}

\author{
Irina Illina \\ Université de Lorraine, CNRS, Inria, Loria \\ F-54000 Nancy, France
}

\begin{abstract}
Speech enhancement promises higher efficiency in ad-hoc microphone arrays than in constrained microphone arrays thanks to the wide spatial coverage of the devices in the acoustic scene. However, speech enhancement in ad-hoc microphone arrays still raises many challenges. In particular, the algorithms should be able to handle a variable number of microphones, as some devices in the array might appear or disappear. In this paper, we propose a solution that can efficiently process the spatial information captured by the different devices of the microphone array, while being robust to a link failure. To do this, we use an attention mechanism in order to put more weight on the relevant signals sent throughout the array and to neglect the redundant or empty channels.
\end{abstract}

Index Terms-Speech enhancement, distributed processing, attention mechanisms, ad-hoc microphone arrays

\section{INTRODUCTION}

Ad-hoc microphone arrays are made of several devices like telephones, tablets or hearing aids, each embedded with one or more microphones. They are usually randomly spread in a room, which brings a wide spatial coverage of the room and thus rich recordings of the acoustic scene. Speech enhancement in ad-hoc microphone arrays can benefit from the increased number of microphones in the array and its wide spatial coverage, but it also raises many challenges. In particular, the limited power and computing capacities of the devices, as well as the unconstrained architecture of the array, make it impossible to rely on a fusion center and impose a distributed processing. Besides, the person carrying one of the devices of the array may come in or leave the area covered by the microphone array. This brings the necessity of a flexible processing that can handle a varying number of

This work was made with the support of the French National Research Agency, in the framework of the project DiSCogs "Distant speech communication with heterogeneous unconstrained microphone arrays" (ANR-17-CE230026-01). Experiments presented in this paper were partially carried out using the Grid5000 testbed, supported by a scientific interest group hosted by Inria and including CNRS, RENATER and several Universities as well as other organizations (see https://www.grid5000). microphones. Solutions based on a classical signal processing approach have been proposed to alleviate the bandwidth or power constraints [1] $-[3]$, and some solutions can be used in arbitrary array topologies [4]-[6]. In recent years, solutions based on deep neural networks (DNNs) have outperformed the signal-based solutions [7]-[9]. However, one drawback of DNNs is that they often require a fixed input dimension, constant at training and testing time, which makes these solutions unflexible to a varying number of channels. A few architectures have been proposed to address the problem of a varying number of microphones. Casebeer et al. for example use recurrent units over the channel axis [10], but this implies that the order of the input channels is relevant, which can't be guaranteed in real scenarios. Other solutions propose to use shared parameters across input channels and to further fuse the different channels [11], [12]. These solutions suffer from the drawback that all channels are considered identically by the neural network, whereas they might contain very different information, especially in the context of ad-hoc microphone arrays where the microphones can be wide apart.

In this paper, we address the typical use case of a person remotely communicating with someone else in a noisy room and recorded by several devices like a telephone or a laptop. Since only the audio signals (and no video) are exchanged between the several devices, we assume that the communication bandwidth is not a limit and that the signals can be exchanged without rate distortion. Rate-constrained speech enhancement in wireless acoustic sensor networks (WASNs) remains an issue, especially with low resource devices like hearing aids [13], [14]. We also assume that the signals are perfectly aligned, although synchronization in WASNs is an open problem [15], [16]. Our main point of concern is to enhance the speech in a manner that does not rely on a fusion center and remains efficient if some of the recording devices disappear, e.g. if one of them shuts down. To do so, we propose a speech enhancement solution that combines classic 
signal processing with DNNs. It processes the information captured over the whole microphone array but limits the number of signals exchanged between nodes and operates in arrays with a varying number of devices. This solution is based on our previous work [17], which benefits from a distributed multichannel Wiener filter (MWF) [1] to alleviate the constraints on the fusion center. It also benefits from the modelling power of DNNs which proved to efficiently use spatial information for a more precise time-frequency (TF) mask estimation. We extend our previous work by designing the DNN so that the mask estimation remains accurate while resilient to a link failure. Missing channels are replaced by a constant value indicating a link failure, and an attention mechanism attributes more weight to the relevant channels. We also design an empirical study to clarify the performance improvement brought by the attention mechanism.

This paper is organised as follows. In Section II] the problem is formalised. We introduce our solution in Section III and the experimental setup in Section IV. The results are reported in Section V] and Section VI concludes this paper.

\section{PROBLEM FORMULATION}

\section{A. Notations}

We consider $K$ devices, thereafter called nodes. Each node $k$ contains $M_{k}$ microphones, so that the total number of microphones is $M=\sum_{k=1}^{K} M_{k}$. Following an additive noise model in the short-time Fourier transform (STFT) domain, the signal recorded by the $m$-th microphone of the $k$-th node is $y_{k, m}(f, t)=s_{k, m}(f, t)+n_{k, m}(f, t)$ where $s_{k, m}(f, t)$ and $n_{k, m}(f, t)$ are respectively the target speech and the noise recorded by the $m$-th microphone of the $k$-th node at time step $t$ and frequency index $f$. For the sake of conciseness, we will thereafter drop the time and frequency indexes. The signals recorded by node $k$ are stacked in a vector $\mathbf{y}_{k}=\left[y_{k, 1}, \ldots, y_{k, M_{k}}\right]^{T}$. In the following, bold lowercase letters represent vectors. Bold uppercase letters represent matrices. Regular lowercase represent scalars. The signals recorded by the whole microphone array are stacked into the vector $\mathbf{y}=\left[\mathbf{y}_{1}^{T}, \ldots, \mathbf{y}_{K}^{T}\right]^{T}$.

\section{B. Distributed multichannel Wiener filter}

Bertrand and Moonen introduced the distributed adaptive node-specific signal estimation (DANSE) algorithm which estimates the target speech as recorded by a reference microphone at each node [1]. At each node, a MWF minimizes the mean squared error between the filtered signal and the target speech:

$$
\mathbf{w}_{k}=\arg \min _{\mathbf{w}} \mathbb{E}\left\{\left|s_{k, m}-\mathbf{w}^{H} \tilde{\mathbf{y}}_{k}\right|^{2}\right\},
$$

$\mathbb{E}\{\cdot\}$ denotes the expectation and $\cdot{ }^{H}$ is the Hermitian transpose operator. $\tilde{\mathbf{y}}_{k}=\left[\mathbf{y}_{k}^{T}, \mathbf{z}_{-k}^{T}\right]^{T}$ gathers the signals of the microphones of node $k$ and the so-called compressed signals $\mathbf{z}_{-k}$ sent by the other nodes: $\mathbf{z}_{-k}=\left[z_{1}, \ldots, z_{k-1}, z_{k+1}, \ldots, z_{K}\right]^{T}$. The compressed signal $z_{j}$ sent by node $j \neq k$ is the output of a local MWF applied at node $j: z_{j}=\mathbf{w}_{j j}^{H} \mathbf{y}_{j}$,

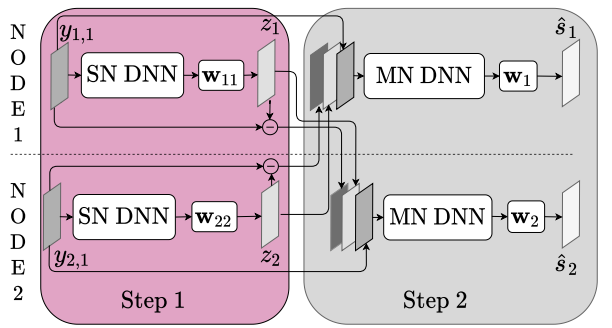

Fig. 1. Example of the DNN-based distributed speech enhancement for two nodes, where both the target and the noise estimates are sent as compressed signals. The single-node DNNs (SN-DNN) have access to the local reference only to predict the mask. The multi-node DNNs (MN-DNN) have access to the local reference and the compressed signals to estimate the mask.

where $\mathbf{w}_{j j}=\arg \min _{\mathbf{w}} \mathbb{E}\left\{\left|s_{j, m}-\mathbf{w}^{H} \mathbf{y}_{j}\right|^{2}\right\}$. The solution to Equation (1) is given by:

$$
\mathbf{w}_{\mathbf{k}}=\mathbf{R}_{\tilde{y} \tilde{y}}^{-1} \mathbf{R}_{\tilde{y} s} \mathbf{e}_{k, m},
$$

where the covariance matrices $\mathbf{R}_{\tilde{y} \tilde{y}}$ and $\mathbf{R}_{\tilde{y} s}$ are estimated from the signals $\tilde{\mathbf{y}}_{k}$ and $s_{k, m}$ thanks to a voice activity detector (VAD) or a TF mask, and where $\mathbf{e}_{k, m}$ is a vector of M-1 zeros and a 1 for to the $m$-th microphone of the $k$-th node.

This algorithm converges to the centralized node-specific MWF, while sparing bandwidth cost as each node sends only one compressed signal to the other nodes [1].

In their paper, Bertrand and Moonen estimate the target speech at node $k$ in an adaptive way where the covariance matrices needed to compute the filter $\mathbf{w}_{k}$ in Equation (2) are estimated by averaging over time the instantaneous spatial covariance matrices. This however raises stability issues that are beyond the scope of this paper. Thus, we split the adaptive process of DANSE into two distinct steps represented in Figure 1. The first step computes the compressed signals and the second step estimates the target speech signal once every node has received the compressed signals of the other nodes.

\section{DNN-based distributed multichannel Wiener filter}

In previous work [18], we replaced the oracle VAD used in DANSE by a TF mask predicted by a convolutional recurrent neural network (CRNN), in a similar manner as [7], [8]. We showed that the compressed signals sent to compute the filter of Equation (2) could also help to improve the mask prediction at the second step by a multi-node DNN. This achieved better performance than with an oracle VAD. In an extended study, we generalized these results to real-life scenarios and showed that sending the noise estimate rather than the target estimate could improve the performance depending on the source to interferences ratio (SIR) at the receiving node [17]. To take full advantage from the spatial coverage of the distributed microphone array, in the following, both the target and noise ${ }^{1}$ estimates will be sent as represented in Figure 1

\footnotetext{
${ }^{1}$ Assuming a noise additive model, the compressed noise $\tilde{n}_{k}$ at node $k$ is estimated as $\tilde{n}_{k}=y_{k, m}-z_{k}$.
} 


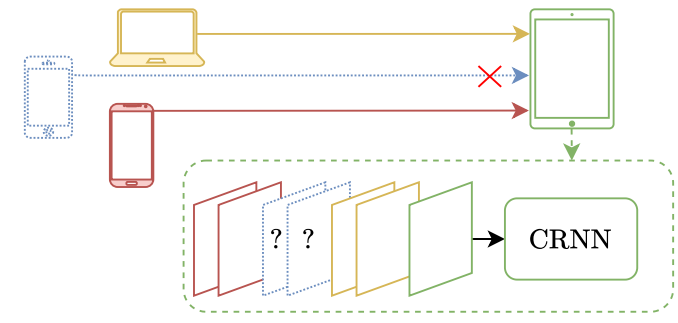

Fig. 2. Schematization of a situation where three nodes are expected to send compressed signals, but only two nodes actually send them. The CRNN expects the local (green) mixture and the target and noise estimates of all distant nodes (yellow, blue, red).

\section{ATtENTION-BASED DistribUtED SPEECH ENHANCEMENT ALGORITHM}

In this paper, we focus on the typical problem of a node disappearing from the microphone array, for example because the owner of the corresponding device leaves the room. Such a case is schematized in Figure 2. The model architecture used in our previous work requires a constant number of input channels so it cannot be used for a variable number of nodes. In the context of broken or disappearing links, this raises an issue which we address in this paper. The contribution of this paper is twofold. First, we propose a solution to deal with a variable number of nodes which is robust to broken links. Second, we examine the performance of this solution with an empirical study in order to explain the obtained performance.

To cope with a variable number of nodes, we fix the number of input channels to a constant maximal number. If a node disappears, we replace the corresponding unreceived signals with a small constant negative value, which symbolises a broken link. While this is limited by the maximal number of devices considered, it seems a valid solution in scenarios where a small number of devices already captures most of the spatial information. We propose to use an attention mechanism to force the DNN to consider differently the input channels. This is illustrated in Figure 3. The attention mechanism is a Squeeze-and-Excitation (SE) block introduced by $\mathrm{Hu}$ et al. [19]. The mechanism operates in two steps. In the first step, it squeezes the input tensor over the time and frequency axis to output a one-dimensional vector. The squeezing operation is an average pooling that enables to compress the whole spatial information into one bin. It embeds the input data into a global vector so that contextual information can be exploited in the second step. In the second step, the one-dimensional vector is passed to a multilayer perceptron composed of two fullyconnected layers. These layers form a bottleneck where the input dimension is reduced by a factor $r$ in order to reduce the complexity of the mechanism and to prevent overfitting. This mechanism was shown to exploit contextual information and dependencies over the channel axis while limiting the complexity of the model [19]. In the sequel, we will refer to the output of the SE mechanism as weights.

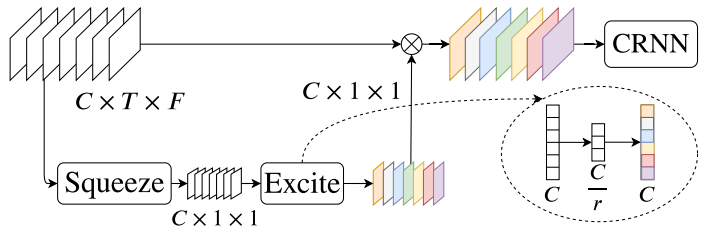

Fig. 3. Illustration of the Squeeze-and-Excitation attention mechanism. $C$, $T$ and $F$ denote the number of channels, time frames and frequency bins respectively. $r$ is the reduction ratio.

\section{EXPERIMENTAL SETUP}

\section{A. Models}

Four models are compared in order to study the performance of our proposed approach. The first model is a single-node CRNN whose structure is described in Section IV-C. It has only access to the local reference signal to estimate the mask at the second step of the algorithm. This is the simplest method that is invariant to the number of nodes, since it does not rely on the compressed signals to predict the mask. It is denoted "SN". The second model is the model that has been used in our previous experiments [17]. It is a multi-node neural network that estimates the mask based on the local reference signal and the compressed signals sent from the other nodes. At inference time, its missing channels are replaced by a constant negative value, but during the training, all the links were always valid. It is denoted by " $\mathrm{MN}_{0}$ ". The third model is the same architecture as the second model, but each training sample could contain 0 to 3 broken links. It is denoted by " $\mathrm{MN}_{0-3}$ ". The fourth model is our proposed solution with a SE mechanism at its input. It is trained with 0 to 3 broken links at every training sample. It is denoted by "MN-SE".

\section{B. Dataset}

The dataset used to train and test our proposed solution is the same as the one of our previous work [17]. It consists of simulations of typical shoebox-like rooms with one target source and one noise source randomly laid in the room. Four nodes of four microphones each are also randomly placed in the room. All sources and nodes are distant of at least $50 \mathrm{~cm}$ of the closest source, node and wall.

The speech material is taken from LibriSpeech [20]. The noise material is downloaded from Freesound [21]. It is split into two non-overlapping subsets of Freesound users for the training and testing sets. Some speech-shaped noise was also used to train the DNN because it was shown to improve the robustness of the DNN [17].

The rooms were simulated with the Python toolbox Pyroomacoustics [22]. The SIR of the non-reverberated source signals is randomly taken between $0 \mathrm{~dB}$ and $6 \mathrm{~dB}$. The reverberation time ranges from $150 \mathrm{~ms}$ to $400 \mathrm{~ms}$. We created around 25 hours of training material and 2.5 hours of testing materia $\sqrt{2}$

\footnotetext{
${ }^{2}$ The code to generate the dataset is publicly available at https://github.com/ nfurnon/disco/tree/master/dataset_generation
} 


\section{Setup}

All the signals are sampled at $16 \mathrm{kHz}$. The STFT is computed with a Hann window of $32 \mathrm{~ms}$ with an overlap of $16 \mathrm{~ms}$. The CRNN architecture is composed of three convolutional layers followed by a recurrent layer and a fully-connected layer. The convolutional layers have 32, 64 and 64 filters, with kernel size $3 \times 3$ and stride $1 \times 1$. Each convolutional layer is followed by a batch normalisation and a maximum-pooling layer of kernel size $4 \times 1$ so that no pooling is applied over the time axis. The recurrent layer is a 256-unit GRU. The fully-connected layer has 257 units with a sigmoid activation function. The reduction ratio in the excitation operation is set to 2. The input of the model are the magnitudes of the STFT windows of 21 consecutive frames and the ground truth labels are the corresponding frames of the ideal ratio mask. At test time, only the middle frame of the predicted window is considered to estimate the mask, so sliding windows of the input are fed to the DNN. The mask of the whole signal is predicted before being used to enhance the speech in a batch mode. When a link is broken between a node and the rest of the array, both the target and the noise magnitudes which are missing are replaced by an array equal to $-10^{-7}$ in all TF bins. This way, the DNN always has 7 input channels (one local signal plus $3 \times 2$ compressed signals) whatever the number of nodes it is connected to.

To better analyse the impact of missing channels on the DNN performance, we only consider missing channels at the input of the DNN and still use all the compressed signals at the filtering operations. This is purely artificial, since available signals at the filtering operations should also be available to predict the mask. However, this setup allows us to disentangle the impact of a broken link on the DNN and on the MWF. We can then analyse the performance from a DNN point of view which is the focus of this paper ${ }^{3}$

\section{Performance evaluation}

Three metrics are used to evaluate the results: the SIR improvement [23], denoted as $\Delta$ SIR; the source to artifacts ratio (SAR); and the short-time objective intelligibility (STOI) improvement [24], denoted as $\Delta$ STOI. The references needed to compute these metrics are the non-reverberated noise and speech signals. All the reported results correspond to the average metric over all the nodes of all configurations of the test set. This was decided in order to report the overall performance in the microphone array.

\section{RESULTS AND ANALYSIS}

\section{A. Resilience to missing channels}

We compare the four models introduced in Section IV-A on the testing set and report the results in Figure 4 Replacing the missing channels by a fixed value can help the DNN to be resilient to link failures, at the condition that this DNN was trained to deal with such signals $\left(\mathrm{MN}_{0-3}, \mathrm{MN}-\mathrm{SE}\right)$. The

\footnotetext{
${ }^{3}$ Preliminary studies showed that missing channels at the filtering operation lead to a limited drop of performance.
}
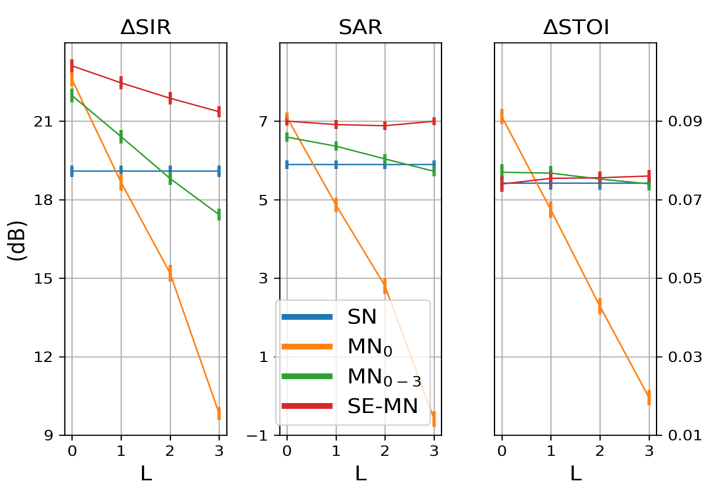

Fig. 4. Performance over link failures. $L$ refers to the number of broken links. The error bars represent the $95 \%$ confidence intervals.

second DNN $\left(\mathrm{MN}_{0}\right)$, which was not trained with broken links, fails to enhance the speech as soon as one of the four nodes is disconnected from the rest of the microphone array. If the DNN is trained to deal with missing channels $\left(\mathrm{MN}_{0-3}\right)$, it can still exploit the spatial information sent from the other nodes since $\mathrm{MN}_{0-3}$ outperforms the single-node DNN when 2 or fewer links are broken. When all nodes are disconnected ( $L=3$ ), the noise reduction is lower than with the singlenode CRNN, because the amount of missing data is too high for the $\mathrm{MN}_{0-3}$. Still, the performance in terms of SAR and STOI is equal between the two models. With an attention mechanism (MN-SE), the noise reduction also decreases when the number of broken links increases, but to a lesser extent than for the other models, and it always significantly outperforms, in terms of SIR and SAR, both the single-node DNN and the multi-node DNN trained with missing channels. Besides, the SAR with MN-SE is constant over $L$, which shows that using this model does not introduce artefacts although channels are missing. This means that the SE mechanism not only helps to exploit the spatial information that is actually received, but also increases the performance of the CRNN even when no compressed signal is received $(L=3)$. Lastly, the increased difference between the performance of $\mathrm{MN}_{0-3}$ and $\mathrm{MN}-\mathrm{SE}$ when $L$ increases indicates a stronger resilience of $\mathrm{MN}-\mathrm{SE}$ compared to the former model.

\section{B. Dissociation of the effects of the attention mechanism}

In this section, we propose an ablation study to disentangle the effects of the SE branch and the effects of the weights. The performance improvement can have two reasons. The first reason is that the weights applied to the channels highlight the channels of interest. The second reason is that the SE branch helps the whole DNN to train better. Considering these two hypotheses, we train the following three DNNs:

- rand-MN; a multi-node CRNN without SE mechanism, and with random weights applied on the input channels.

- SE-rand-MN; a multi-node CRNN with SE mechanism but whose attention weights are replaced by random values at train time and at inference time. 

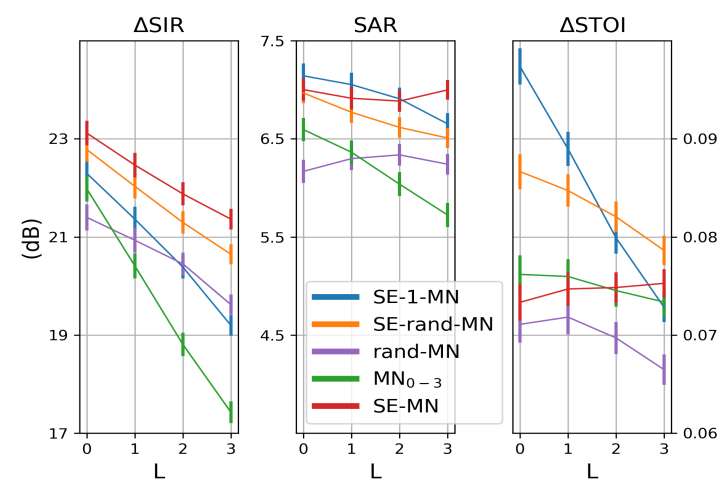

Fig. 5. Performance over link failures when the effects of the SE mechanism and of the weights are dissociated. $L$ refers to the number of broken links. The error bars represent the $95 \%$ confidence intervals.

- SE-1-MN; a multi-node CRNN with SE mechanism but whose attention weights are replaced by 1 at train time and at inference time.

The results of these models, together with the results of the previous models $\mathrm{MN}-\mathrm{SE}$ and $\mathrm{MN}_{0-3}$, are represented in Figure 5. The impact of the SE module alone can be studied by comparing MN-SE-rand with MN-rand and MN-SE with $\mathrm{MN}_{0-3}$. In both cases the SE module helps improving the overall performance and the robustness of the model. The impact of the weights alone is more complex to analyse. Comparing MN-rand with $\mathrm{MN}_{0-3}$ and MN-SE-rand with MN-SE-1 helps understanding the influence of applying any weights on the input of the CRNN. Although this brings worse performance in terms of STOI, it increases the robustness of the models. Indeed, when $L$ increases, the performance of the models with weights decreases much less than the performance of the models without weights. Lastly, comparing MN-SE-1 with MN-SE helps analysing the influence of applying the correct weights on the input of the CRNN. From the results, using the correct weights increases the SIR and SAR but lowers the STOI. However, the model without weights is much less robust to missing channels, as the STOI decreases drastically when $L$ increases. To sum up, the SE branch helps increasing the performance of the whole model, even with random weights applied on the input of the CRNN. Using the correct values of the weights is important primarily for a higher number of missing channels.

\section{CONCLUSION}

We introduced a distributed multichannel speech enhancement algorithm that handles a varying number of input channels. Based on an attention mechanism, it exploits the spatial information and minimizes the performance drop due to link failures. An ablation study led to the conclusion that the SE mechanism helps to improve the performance of the whole network, and that the weights importance increases with the number of missing channels. Future works foresees to analyse the behaviour of the proposed system when the assumptions about the bit-rate and synchronization do not hold.

\section{REFERENCES}

[1] A. Bertrand and M. Moonen, "Distributed adaptive node-specific signal estimation in fully connected sensor networks - Part I: Sequential node updating," IEEE Transactions on Signal Processing, vol. 58, no. 10, pp. 5277-5291, Oct 2010.

[2] R. Heusdens, G. Zhang, R. C. Hendriks, Y. Zeng, and W. B. Kleijn, "Distributed MVDR beamforming for (wireless) microphone networks using message passing," IWAENC, pp. 1-4, 2012.

[3] M. O'Connor and W. B. Kleijn, "Diffusion-based distributed MVDR beamformer," IEEE ICASSP, pp. 810-814, 2014.

[4] J. Szurley, A. Bertrand, and M. Moonen, "Topology-independent distributed adaptive node-specific signal estimation in wireless sensor networks," IEEE TSIPN, vol. 3, no. 1, pp. 130-144, 2016.

[5] A. I. Koutrouvelis, T. W. Sherson, R. Heusdens, and R. C. Hendriks, "A low-cost robust distributed linearly constrained beamformer for wireless acoustic sensor networks with arbitrary topology," IEEE/ACM TASLP, vol. 26, no. 8, pp. 1434-1448, 2018.

[6] X. Guo, M. Yuan, C. Zheng, and X. Li, "Distributed node-specific blockdiagonal LCMV beamforming in wireless acoustic sensor networks," arXiv preprint arXiv:2010.13334, 2020.

[7] H. Erdogan, T. Hayashi, J. R. Hershey, T. Hori, C. Hori, W. Hsu, S. Kim, J. Le Roux, Z. Meng, and S. Watanabe, "Multi-channel speech recognition: LSTMs all the way through," CHiME-4, pp. 1-4, 2016.

[8] J. Heymann, L. Drude, and R. Haeb-Umbach, "Neural network based spectral mask estimation for acoustic beamforming," IEEE ICASSP, pp. 196-200, 2016.

[9] N. Tawara, T. Kobayashi, and T. Ogawa, "Multi-channel speech enhancement using time-domain convolutional denoising autoencoder.," INTERSPEECH, pp. 86-90, 2019.

[10] J. Casebeer, B. Luc, and P. Smaragdis, "Multi-view networks for denoising of arbitrary numbers of channels," IWAENC), pp. 496-500, 2018.

[11] Y. Luo, Z. Chen, N. Mesgarani, and T. Yoshioka, "End-to-end microphone permutation and number invariant multi-channel speech separation," IEEE ICASSP, pp. 6394-6398, 2020.

[12] D. Wang, Z. Chen, and T. Yoshioka, "Neural speech separation using spatially distributed microphones," arXiv preprint arXiv:2004.13670, 2020.

[13] O. Roy and M. Vetterli, "Rate-constrained collaborative noise reduction for wireless hearing aids," IEEE Transactions on Signal Processing, vol. 57, no. 2, pp. 645-657, 2008.

[14] J. Amini, R. C. Hendriks, R. Heusdens, M. Guo, and J. Jensen, "Rate-constrained noise reduction in wireless acoustic sensor networks," IEEE/ACM TASLP, vol. 28, pp. 1-12, 2020.

[15] J. Schmalenstroeer, P. Jebramcik, and R. Haeb-Umbach, "A combined hardware-software approach for acoustic sensor network synchronization," Signal Processing, vol. 107, pp. 171-184, 2015.

[16] A. Chinaev, P. Thuene, and G. Enzner, "Double-cross-correlation processing for blind sampling-rate and time-offset estimation," IEEE/ACM TASLP, 2021.

[17] N. Furnon, R. Serizel, I. Illina, and S. Essid, "DNN-based mask estimation for distributed speech enhancement in spatially unconstrained microphone arrays," arXiv preprint arXiv:2011.01714, 2020.

[18] N. Furnon, R. Serizel, I. Illina, and S. Essid, "DNN-based distributed multichannel mask estimation for speech enhancement in microphone arrays," IEEE ICASSP, pp. 4672-4676, 2020.

[19] J. Hu, L. Shen, and G. Sun, "Squeeze-and-excitation networks," Proceedings of the IEEE conference on computer vision and pattern recognition, pp. 7132-7141, 2018.

[20] V. Panayotov, G. Chen, D. Povey, and S. Khudanpur, "Librispeech: an ASR corpus based on public domain audio books," IEEE ICASSP, pp. 5206-5210, 2015.

[21] F. Font, G. Roma, and X. Serra, "Freesound technical demo," $A C M$ International Conference on Multimedia (MM'13), pp. 411-412, 2013.

[22] R. Scheibler, E. Bezzam, and I. Dokmanic, "Pyroomacoustics: A python package for audio room simulation and array processing algorithms," IEEE ICASSP, Apr 2018

[23] E. Vincent, R. Gribonval, and C. Févotte, "Performance measurement in blind audio source separation," IEEE TASLP, vol. 14, no. 4, pp. 1462-1469, 2006.

[24] C. H. Taal, R. C. Hendriks, R. Heusdens, and J. Jensen, "A shorttime objective intelligibility measure for time-frequency weighted noisy speech," IEEE ICASSP, pp. 4214-4217, 2010. 